\title{
QUADRANTID METEOR SHOWER: ELEVEN YEARS OF RADAR OBSERVATIONS
}

\author{
B. A. MCINTOSH
}

In studying the evolution of meteor streams from cometary debris it is desirable to know the distribution of particles both around the orbit and in the cross section perpendicular to the orbit. Earth-based observations are limited to a once-per-year sample and also limited by the position of the observer on earth as it crosses the meteor stream. A continuous series, 11 years, of records of the Quadrantid meteor shower illustrates the problems and the results that can be achieved. International cooperation would greatly assist such studies.

The solid particles left as the debris of a comet eventually distribute themselves into a more or less continuous belt around the orbit. In considering the evolution of such a stream it is desirable to know in some detail the particle density around the orbit and across the orbit, and to what extent the distributions vary with particle size.

The earth is a rather poor sampling probe permitting only one sample per year of a meteor shower. Between annual samples one does not really know the nature of the particle distribution. Further, because the earth rotates, a narrow shower may not be well observed if the observer happens to be on the back side of the earth as it passes through the core of the stream.

One such stream of considerable interest is the Quadrantid meteor shower which occurs about January 3. The comet which originated the shower is not known. Yet it is a very young stream with a high proportion of small particles and a very narrow width. Because its orbit is fairly smal1, with a period of about 5 years, there is a possibility of accumulating enough annual samples to look at the distribution of particles around the orbit. At Ottawa, Canada, on January 3 rd it is usually $-25^{\circ} \mathrm{C}$ and/or cloudy, so one has to rely on radar observations for a continuous series of samples.

The stream's narrow width makes determination of the flux through the stream very difficult because the radar sensitivty is a complex function of the position angle between the observer on the earth and the shower radiant point on the celestial sphere (McIntosh 1966). A semi-empirical method has been used to correct observed radar echo counts to relative meteor flux values. Determination of the time (solar longitude) of the occurrence of peak flux is shown in Fig. 1. The bars represent the width of the flux curves at $60 \%$ of the maximum flux. It is apparent that in some years, 1965 and 66 in particular, the flux curve was very narrow at the core and the location of maximum particle density was well determined but significantly different from year to year. In 
MC INTOSH

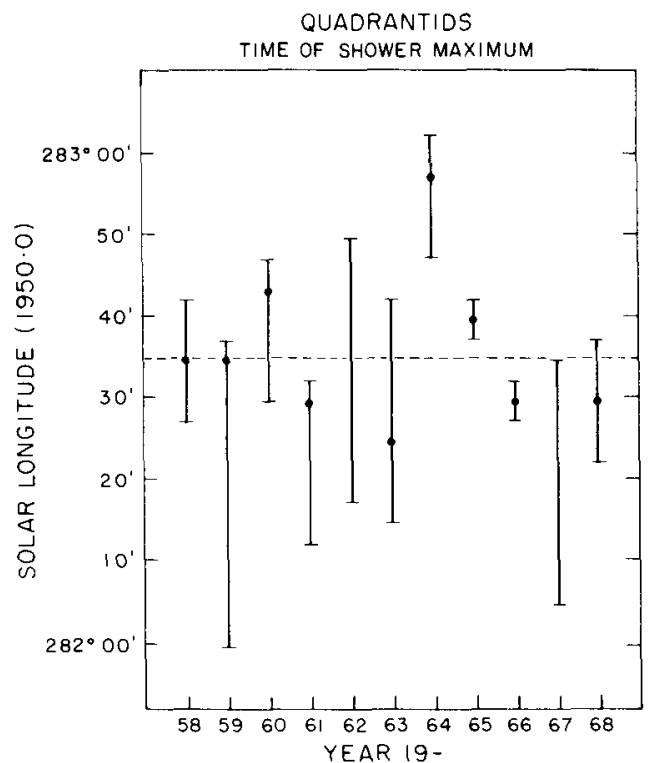

Figure 1 . Solar longitude of maximum flux of the Quandrantid meteor shower from eleven years of radar observations.

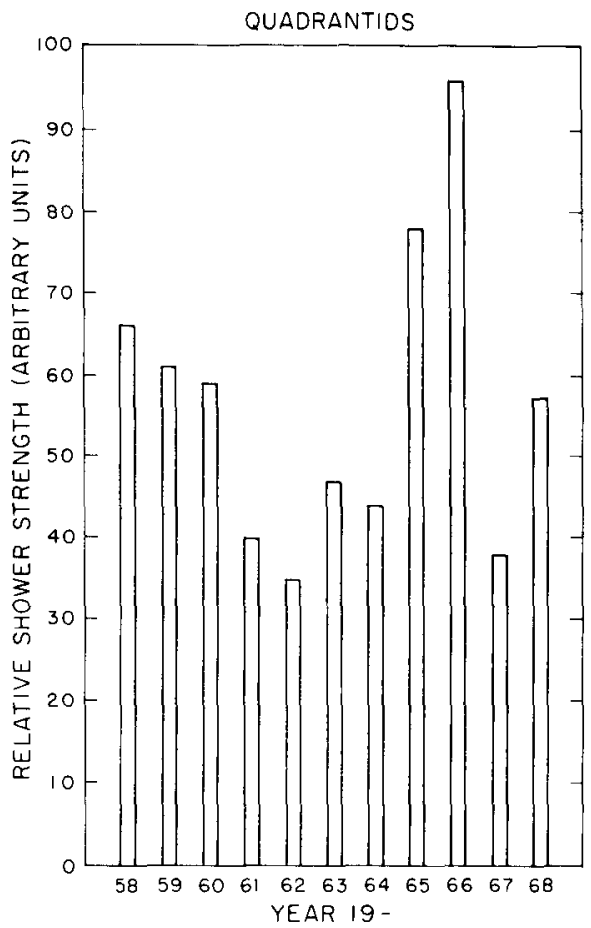

Figure 2. Relative strength of the Quandrantid meteor shower 1958-1968. 
other years the flux curve was so broad and flat that a specific time of maximum could not be determined.

A mean value at solar longitude $282.51^{\circ}$ is very close to that determined from other radar observations (Poole et al. 1972). Maxima for the last 3 years individually agree closely with values determined by Belkovitch (1974). A mean position from visual data given by Hindley (1972) is about $282.8^{\circ}$ indicating some sorting of particles, the larger particles peaking later than the small ones.

In Fig. 2 are shown relative flux rates for the eleven years. One expects to see the periodicity of the orbit reflected in the particle density distribution as in the case of the Geminid meteor shower (Hajduk et al. 1974; McIntosh 1974) but there is no obvious pattern here. The peak years may be only isolated concentrations.

Because the aphelion of the stream orbit lies near the orbit of Jupiter, this shower offers an opportunity to study the effects on meteor streams of planetary perturbations. Some of the flux variations shown in Fig. 2 may in fact be due to Jovian perturbations.

It is believed that this meteor shower merits further long-term study. International cooperation to provide simultaneous observations at about 3 stations well spaced around the earth would be of great benefit in such research since it would eliminate the problem of possible poor flux determination in some years.

\section{REFERENCES}

Belkovich, D. I., and Tohktasev, V. S. 1974, Bull. Astron. Inst. Czech., 25, 370 .

Hajduk, A., McIntosh, B. A., and Simek, M. 1974, Bull. Astron. Inst. Czech., 25,305 .

Hindley, K. B. 1972, Sky and Telescope, 44, 162.

McIntosh, B. A. 1966, Can. J. Phys., 44, 2729.

McIntosh, B. A. 1971, Bul1. Astron. Inst. Czech., 25, 362.

Poole, L.M.G., Hughes, D. W., and Kaiser, T. R. 1972, Mon. Not. R. Astr. Soc., 156,223 .

\section{DISCUSSION}

HUGHES: Two small comments. Firstly atmospheric effects (e.g., variation of mesopause temperature in January with phase in the solar cycle) will have a very small effect on the observed flux (less than $10 \%$ ).

second, it must be remembered that Jupiter passes the aphelion area of the Quadrantids about every 12 years when it perturbs about $10 \%$ of the particles in the stream, the effect of the perturbation being detected at earth about two years later when these meteoroids have come around the orbit to about I AU. This tends to change the mean ascending node (shown in your lst slide) every 12 years.

KEAY: 1) Does the coincidence between the 1965-66 years of maximum flux and minimum stream width imply that this part of the orbit is where the parent body lies? 2) But the lack of similarity with 5 years previously simply means that 
the intersection with the Earth's orbit has varied to a different part of the stream cross-section. Can you get a clue to the direction of the parent from this?

MCINTOSH: One of the interesting aspects of the Quadrantid shower is that its aphelion is in the vicinity of Jupiter's orbit. Peak rates such as those of 1965 and 1966 may be natural concentrations in the stream or they may be due to Jupiter perturbations. Without a more extensive series of measurements I really don't know which. The widths indicated are those at about $60 \%$ of the peak rate. The widths down to near zero rate do not vary very much. 